\title{
Chemoinformatic Analysis of Psychotropic and Antihistaminic Drugs in the Light of Experimental Anti-SARS-CoV-2 Activities
}

This article was published in the following Dove Press journal:

Advances and Applications in Bioinformatics and Chemistry

\author{
Bruno O Villoutreix (iD) \\ Rajagopal Krishnamoorthy $\mathbb{( D}^{2}$ \\ Ryad Tamouza ${ }^{2}$ \\ Marion Leboyer (D) $^{2}$ \\ Philippe Beaune ${ }^{3}$ \\ 'INSERM UI I4I, NeuroDiderot,
} Université de Paris, Hôpital Robert-Debré, Paris, F-750I 9, France; ${ }^{2}$ Université Paris Est Créteil, INSERM U955, IMRB, Laboratoire Neuropsychiatrie Translationnelle, AP-HP, Département Medico-Universitaire de Psychiatrie et d'Addictologie (DMU ADAPT), Hôpital Henri Mondor, Fondation FondaMental, Créteil, F-940I0, France; ${ }^{3}$ INSERM UI I 38, Centre de Recherche des Cordeliers, Université de Paris, Paris, 75006, France

Correspondence: Bruno O Villoutreix Email bruno.villoutreix@inserm.fr
Introduction: There is an urgent need to identify therapies that prevent SARS-CoV-2 infection and improve the outcome of COVID-19 patients.

Objective: Based upon clinical observations, we proposed that some psychotropic and antihistaminic drugs could protect psychiatric patients from SARS-CoV-2 infection. This observation is investigated in the light of experimental in vitro data on SARS-CoV-2.

Methods: SARS-CoV-2 high-throughput screening results are available at the NCATS COVID-19 portal. We investigated the in vitro anti-viral activity of many psychotropic and antihistaminic drugs using chemoinformatics approaches.

Results and Discussion: We analyze our clinical observations in the light of SARS-CoV-2 experimental screening results and propose that several cationic amphiphilic psychotropic and antihistaminic drugs could protect people from SARS-CoV-2 infection; some of these molecules have very limited adverse effects and could be used as prophylactic drugs. Other cationic amphiphilic drugs used in other disease areas are also highlighted. Recent analyses of patient electronic health records reported by several research groups indicate that some of these molecules could be of interest at different stages of the disease progression. In addition, recently reported drug combination studies further suggest that it might be valuable to associate several cationic amphiphilic drugs. Taken together, these observations underline the need for clinical trials to fully evaluate the potentials of these molecules, some fitting in the so-called category of broad-spectrum antiviral agents. Repositioning orally available drugs that have moderate side effects and should act on molecular mechanisms less prone to drug resistance would indeed be of utmost importance to deal with COVID-19.

Keywords: chemoinformatics, COVID-19, high-throughput screening, phenothiazine, prophylaxis, antihistamine, drug repurposing

\section{Introduction}

The rapid pandemic spread of SARS-CoV-2 virus and the resulting COVID-19 has caused havoc worldwide (with millions of deaths, https://www.worldometers.info/ coronavirus/) and continue to do so with transmission rate spikes. Hence, there is an urgent need to identify potential pharmacological prophylactic/therapeutic interventions both to prevent SARS-CoV-2 infection and to improve the clinical outcome of COVID-19 patients. At present, only vaccines offer hope but small molecules interfering with SARS-CoV-2 infection would have an added value and could be cost-effective. The shortest path to discover such compounds is via drug repurposing approaches but it should be mentioned, as reported by Edwards, ${ }^{1}$ that drug 
repurposing seldom works in humans if there are no clinical observations as background rationale and/or in the absence of a reasonable mechanistic hypothesis. Early 2020, we hypothesized, based upon clinical observations made in several hospitals including the large psychiatric department of the Henri Mondor hospital, Créteil (Paris area), France, and confirmed in several other hospitals, that therapeutic molecules used in the treatment of psychiatric patients might have protected them from SARSCoV-2 infection and hence explained the paucity of admission to specialized COVID-19 units. $^{2-5}$ In order to gain knowledge about the putative molecules that could be repositioned against SARS-CoV-2, we collected a list of 18 compounds most commonly prescribed in the in- and out-patient Henri Mondor treatment programs (Alimemazine, Amisulpride, Aripiprazole, Cetirizine, Citalopram, Clozapine, Cyamemazine, Diazepam, Escitalopram, Hydroxyzine, Lithium, Lorazepam, Melatonin, Nicotine, Quetiapine, Sertraline, Valproate, and Zopiclone). Some of these compounds belong to the phenothiazine class (eg, Alimemazine and Cyamemazine), others contain a benzodiazepine core (eg, Lorazepam) or else are antihistamine drugs with, for example, a core piperazine group (eg, Cetirizine and Hydroxyzine). We investigated further these 18 drugs using chemoinformatic strategies and via literature mining as it is well documented that some molecules from these classes possess in vitro antiviral activities (reviewed $\mathrm{in}^{6,7}$ ). Indeed, 10 out of our 18 drugs have been reported to have in vitro antiviral activities against various types of viruses including HIV, Ebola, HSV, MERS or SARS. Four were chemically close to molecules that display such in vitro antiviral activity and were therefore predicted to also possess such property based on "principle of similarity" commonly applied in medicinal chemistry. ${ }^{8}$ While the psychotropic and antihistamine drugs that we were investigating belong to several chemical families, we observed a common trend among these molecules. Eleven (60\%) out of these 18 most prescribed compounds were cationic amphiphilic drugs (CADs). CADs are molecules characterized by the presence of a hydrophobic-aromatic ring system and a side chain that carries one (or more) ionizable amine functional group(s) (ie, a basic moiety). Many such compounds accumulate in lysosomes or in other acidic subcellular compartments (ie, lysosomotropic agents, ${ }^{9}$ well-known molecules acting on SARS-CoV-2 in vitro via this mechanism are the antimalarial drugs, chloroquine and hydroxychloroquine although the compounds have also many other actions ${ }^{10-13}$ ). Many CADs are therefore known to interfere with intracellular trafficking (eg, endosomal entry route, clathrin-mediated or not) and several such molecules have indeed in vitro antiviral activity. ${ }^{7,14}$ CADs compounds are also known to induce phospholipidosis in vitro (a lysosomal storage disorder), and 14 out of our list of 18 drugs were known or predicted to induce phospholipidosis. ${ }^{15}$ These molecules are associated with intracellular traffic perturbations and could play a role in SARS-CoV-2 infectivity. ${ }^{16}$ The perturbation of lysosomal function seems to confer anticancer activity to several CAD psychotropic drugs. ${ }^{17}$

From these observations, presumably, many of our 18 molecules could play a role in the early phases of the virus life cycle. In short, in early 2020, our hypothesis was that interesting compounds to reposition against SARS-CoV-2 would be CAD psychotropic and antihistaminic molecules (ie, the ones present in our list of 18 compounds or analogs that possess similar physicochemical properties and chemical substructures). These drugs could perturb virus entry, for instance by interfering with normal endocytosis but could also inhibit directly or indirectly the interaction between the viral Spike protein and the host ACE2 receptor (details about cell entry mechanisms are reported here ${ }^{18}$ ). These drugs obviously bind to their primary targets (for example, histamine receptors, dopamine receptors, GABA(A) receptor), although the impact of such interactions on COVID-19 is not clear. In addition, it is well known that drugs can interact with several targets and as such, the CAD psychotropic and antihistaminic molecules are likely to also bind to known (eg, the Sigma receptors ${ }^{19}$ ) and unknown off-targets and act on the virus and/or on the host defense mechanisms. The conclusion of our first analysis was that CAD psychotropic and antihistaminic molecules could confer protection against SARS-CoV-2 infection in psychiatric patients as these subjects receive as treatment a "CAD cocktail" that certainly interferes with the endo-lysosomal viral machinery essential for cell entry, replication and spread to other cells ${ }^{2}$ while some compounds (eg, antihistamine drugs) could also down-regulate prominent inflammatory messengers. ${ }^{14,20}$ Yet, in our first report, we also mentioned that while such drugs might protect against SARS-CoV-2 infection, many of them do have concerns (eg, toxic side effects if unmonitored) and hence cannot be recommended for mass population prophylaxis and/or treatment in the urgent fight against COVID-19.

When we reported our study about the possible protective potentials of psychotropic and antihistamine drugs 
against SARS-CoV-2 infection, ${ }^{2}$ in vitro data on SARS$\mathrm{CoV}-2$ were not available. Since then, low-throughput and high-throughput screening experiments have been performed (see a recent review ${ }^{21}$ ). To the best of our knowledge, the largest high-throughput screening data open to the research/medical community are available at the NCATS COVID-19 portal (https://opendata.ncats.nih.gov/ $\underline{\text { covid19) }} .{ }^{22}$ These data involve the screening of over 10,000 chemical compounds. We here investigate our initial observations in the light of these new experimental data. We thus further rationalize the potential protective effect of some psychotropic and antihistamine drugs against SARS-CoV-2 infection and propose some already approved drugs (with manageable/tolerable adverse effects) that could be repositioned to protect the general population against COVID-19.

\section{Methods \\ Data Preparation and Analysis}

The first step involved the curation and preparation of the experimental screening data. We downloaded the data on SARS-CoV-2 cytopathic effect (CPE) from the NCATS COVID-19 portal. This assay measures the ability of a compound to reverse the cytopathic effect induced by the virus in Vero E6 host cells (one of the most commonly used cell lines for studying this family of virus). The assay provides valuable basic information about the ability of a compound to restore the cells' function but does not provide indication of the possible mechanism of action(s) of the small chemical compound under study. We also explored using the same Vero E6 cells the cytotoxic effect of these molecules in a counterscreen since the observed effect of some compounds may be mediated through cell viability and could lead to erroneous interpretation of the CPE results. Such information on cell viability is evidently important in the applicability of such compounds in clinical pharmacological interventions. Some additional data are important to take into account in the present analysis and involve the effect of the chemical compounds on human fibroblasts. This assay measures the host cell ATP content as a readout for cytotoxicity. When available, we annotated further the CPE data with the results of this assay. To be able to quickly repurpose a compound, we focused essentially on molecules that have been approved or are in clinical trials (in some instances, drugs are only approved for veterinary use). We thus cross-linked the CPE sample ID numbers to drug names (eg, international nonproprietary names), PubChem SID or CID numbers ${ }^{23}$ or to ChEMBL ID numbers ${ }^{24}$ and to molecules that possess ATC (Anatomical Therapeutic Chemical Classification system) codes and/or have documented therapeutic indications as found in DrugBank, ${ }^{25}$ DrugCentral $^{26}$ and eDrug3d. ${ }^{27}$ Molecules that are at the preclinical stage (eg, chemical probes) were essentially excluded via this procedure. Additionally, the salts, when present in the chemical file, were deleted using the Maya chemistry toolkit, ${ }^{28}$ and the chemistry of the molecules was standardized using our FAF-Drugs 4 web server. ${ }^{29}$ The drugs in duplicate were removed. Further curation of the CPE data also involved the following: i) removal of a few molecules without potency values (AC50 values are reported for this dataset, ie, concentration for half-maximal activity ${ }^{30}$ ), ii) investigation of the curve class (ie, the evaluation of the quality of the dose-response curve and thus the quality of the measurements) in the CPE data (a curve class of type 4 is a flat curve indicating no activity) and maximum response (ie, maximum response value detected in the experiment). In such assay, compounds are usually considered active when the maximum response (absolute) value is above $30 .{ }^{31}$ Further, we removed molecules that were found highly cytotoxic in the Vero E6 counterscreen assay. From the initial CPE data containing 10,721 assessed samples (some molecules have been screened several times and most are chemical probes), we ended up with a list of 198 bioactive drugs. In this list of drugs, 93 were not tested for toxicity on human fibroblast and for the remaining 105 molecules, only one was found cytotoxic (Disulfiram, used for alcohol addiction, this molecule was also removed not only because of its cytotoxic nature in these assays but also as found to be a promiscuous SARS-CoV-2 main protease inhibitor ${ }^{32}$ ). At this stage of the analysis, we decided to retain all the compounds including those not annotated for human fibroblast toxicity. We then focused our attention on families of molecules that contain several members and removed some molecules with highly specific disease indication such as Deferasirox (an oral iron chelator given to patients receiving long-term blood transfusions). In this last step of data curation, we grouped the 198 compounds according to chemistry and disease indications and removed molecules that did not fulfill our selection criteria. This step led to the selection of a final list of 161 molecules reported in Table 1. Numerous approaches can be used to investigate low molecular weight chemical compounds in silico $\left(\mathrm{eg},{ }^{33-35}\right)$ and here we decided to analyze these molecules 
Table I Investigated Chemical Probes and Approved Drugs



(Continued) 
Table I (Continued).

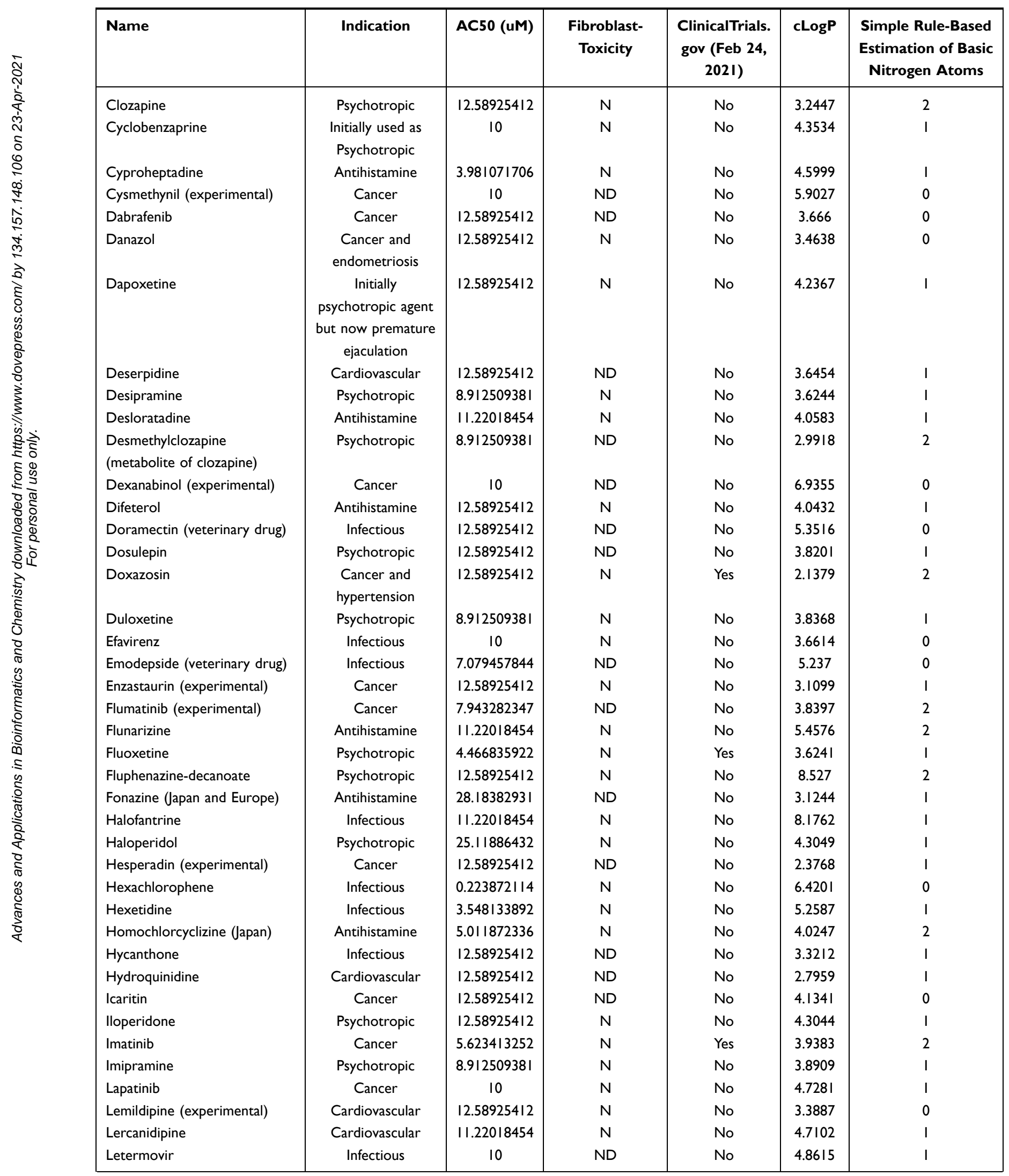


Table I (Continued).

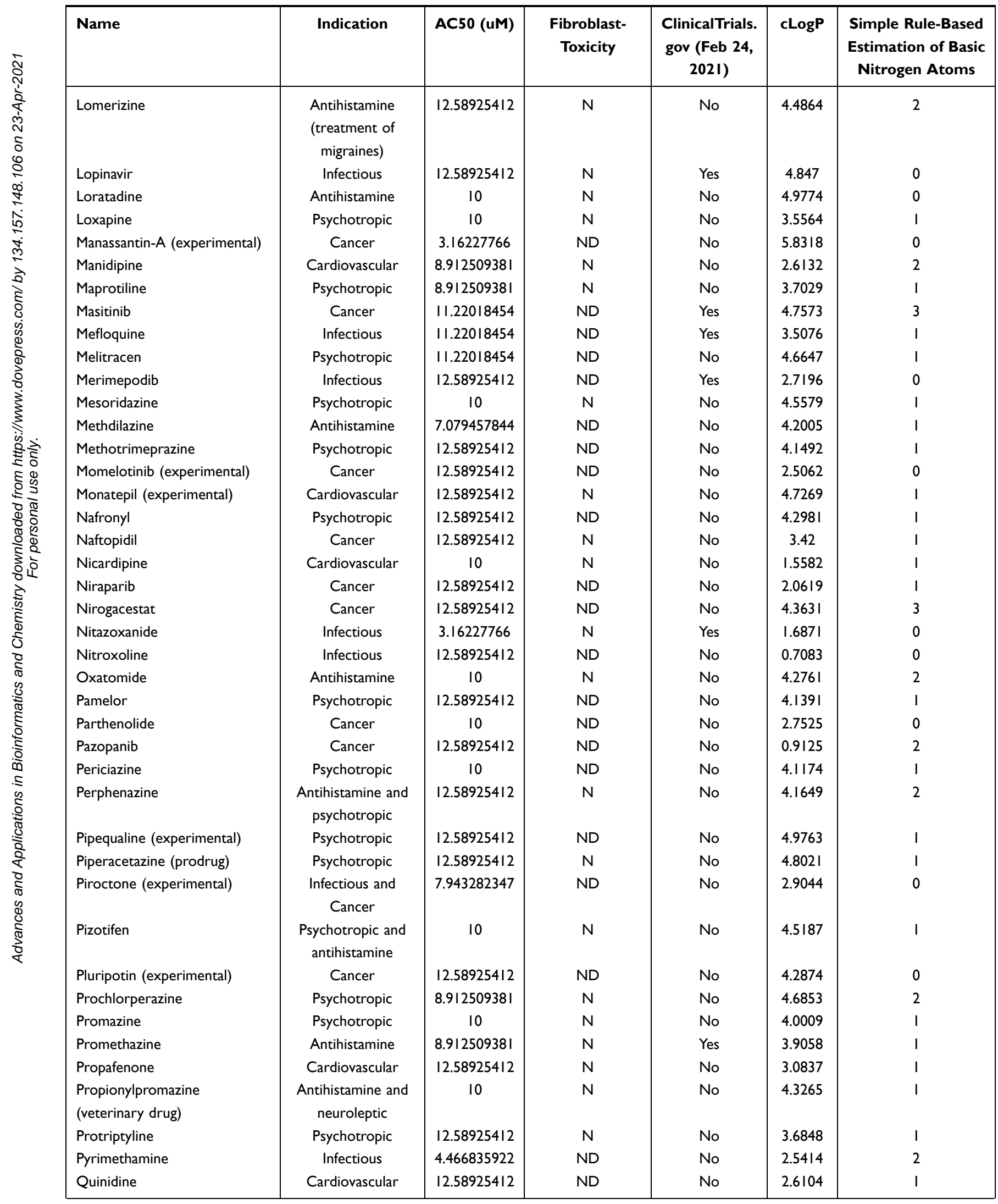


Table I (Continued).

\begin{tabular}{|c|c|c|c|c|c|c|}
\hline Name & Indication & AC50 (uM) & $\begin{array}{l}\text { Fibroblast- } \\
\text { Toxicity }\end{array}$ & $\begin{array}{c}\text { ClinicalTrials. } \\
\text { gov (Feb 24, } \\
2021 \text { ) }\end{array}$ & cLogP & $\begin{array}{c}\text { Simple Rule-Based } \\
\text { Estimation of Basic } \\
\text { Nitrogen Atoms }\end{array}$ \\
\hline Quizartinib (experimental) & Cancer & 2.511886432 & ND & No & 4.7979 & 2 \\
\hline Refametinib (experimental) & Cancer & 11.22018454 & ND & No & 1.88 & 0 \\
\hline Remdesivir & Infectious & 7.943282347 & ND & Yes & 0.3048 & 0 \\
\hline Rescimetol (Japan) & Cardiovascular & 12.58925412 & $\mathrm{~N}$ & No & 3.6989 & 1 \\
\hline Reserpine & Cardiovascular & II.220|8454 & $N$ & Yes & 3.5754 & 1 \\
\hline Retapamulin & Infectious & 12.58925412 & ND & No & 5.218 & 1 \\
\hline Sarpogrelate & Cardiovascular & $12.589254 \mid 2$ & $N$ & No & 1.8567 & 1 \\
\hline Serdemetan (experimental) & Cancer & 8.912509381 & ND & No & 3.7237 & 1 \\
\hline Siccanin (experimental) & Infectious & 12.58925412 & ND & No & 4.3637 & 0 \\
\hline Spiclomazine (experimental) & Cancer & $12.589254 \mid 2$ & ND & No & 5.2169 & 1 \\
\hline Spiperone (Japan) & Psychotropic & 7.943282347 & $N$ & No & 3.0219 & 1 \\
\hline Spiramycin-II & Infectious & $12.589254 \mid 2$ & $N$ & No & 2.4608 & 2 \\
\hline Sulfatinib (experimental) & Cancer & $12.589254 \mid 2$ & ND & No & 2.0918 & I \\
\hline Teicoplanin & Infectious & 14.12537545 & ND & Yes & -3.4194 & 1 \\
\hline Thioproperazine & Psychotropic & II.220I8454 & $N$ & No & 3.2979 & 2 \\
\hline Thiothixene & Psychotropic & 12.58925412 & ND & No & 2.937 & 2 \\
\hline Tilorone & Infectious & 10 & ND & No & 4.0607 & 2 \\
\hline Timiperone (Japan) & Psychotropic & 10 & ND & No & 3.7407 & 1 \\
\hline Tioguanine & Cancer & 10 & ND & No & -0.9683 & I \\
\hline Tipifarnib (experimental) & Cancer & $12.589254 \mid 2$ & ND & No & 4.03 & 2 \\
\hline Tivantinib (experimental) & Cancer & $12.589254 \mid 2$ & ND & No & 4.1837 & 0 \\
\hline Tizoxanide & Infectious & 3.16227766 & ND & Yes & 1.3547 & 0 \\
\hline Triamterene & Cardiovascular & 7.079457844 & $N$ & Yes & 0.6075 & 2 \\
\hline Trifluomeprazine & Psychotropic & 10 & ND & No & 5.0675 & 1 \\
\hline Trifluoperazine & Psychotropic & $|2.589254| 2$ & ND & No & 4.9276 & 2 \\
\hline Triflupromazine & Psychotropic & $11.220 \mid 8454$ & $N$ & No & 4.8492 & 1 \\
\hline Trimeprazine (or Alimemazine) & Psychotropic & 8.912509381 & $N$ & No & 4.2192 & 1 \\
\hline Trimetrexate & Cancer & 10 & ND & No & 1.9786 & 2 \\
\hline Trimipramine & Psychotropic & 10 & $N$ & No & 4.1092 & 1 \\
\hline Vilazodone & Psychotropic & 10 & ND & No & 3.6629 & 1 \\
\hline Vorapaxar & Cardiovascular & $8.9|250938|$ & ND & No & 4.7865 & 0 \\
\hline Zotepine & Psychotropic & $12.589254 \mid 2$ & $N$ & No & 4.6741 & 1 \\
\hline
\end{tabular}

Notes: The names of the $16 \mathrm{I}$ selected bioactive molecules are reported in the first column. For each compound, the main indication, the experimental AC50 values (see text), the experimental fibroblast toxicity (ND, not determined, N, No), the computed log P (cLogP), and a simple estimation of the number of basic nitrogen atoms are shown. Also, molecules that are in clinical trials for COVID-19 treatments indicated; Experimental and investigational drugs are all labelled in this Table experimental.

with DataWarrior. ${ }^{36}$ In this final list, very few molecules are only chemical probes, like Calpeptin (moderate activity against SARS-CoV-2 main protease $\left.\mathrm{M}^{\text {pro }}\right)$. They were kept because they are often used to assess some enzymatic activities. The structural classification of chemical entities was then performed using the ClassyFire application. ${ }^{37}$

\section{Results and Discussion}

The 161 molecules found to be active in the CPE assay (after excluding compounds that were found to be highly cytotoxic in Vero E6 cells counterscreen assay) are prescribed as treatments in five major disease areas (Figures 1 and 2). Of course, it is important to note that some of the selected drugs can be given to different types of disease but we still attempted to group them into main/major indications.

Molecules that are in the "Allergies" category (different types of allergies are involved) are here all antihistamine compounds. Some of these molecules also have other properties, such as anti-inflammatory effects. This group of compounds includes for instance Loratadine and Desloratadine (eg, allergic rhinitis), Azelastine (eg, allergic and vasomotor rhinitis and allergic conjunctivitis) and Promethazine (eg, used for allergic reactions, nausea and 


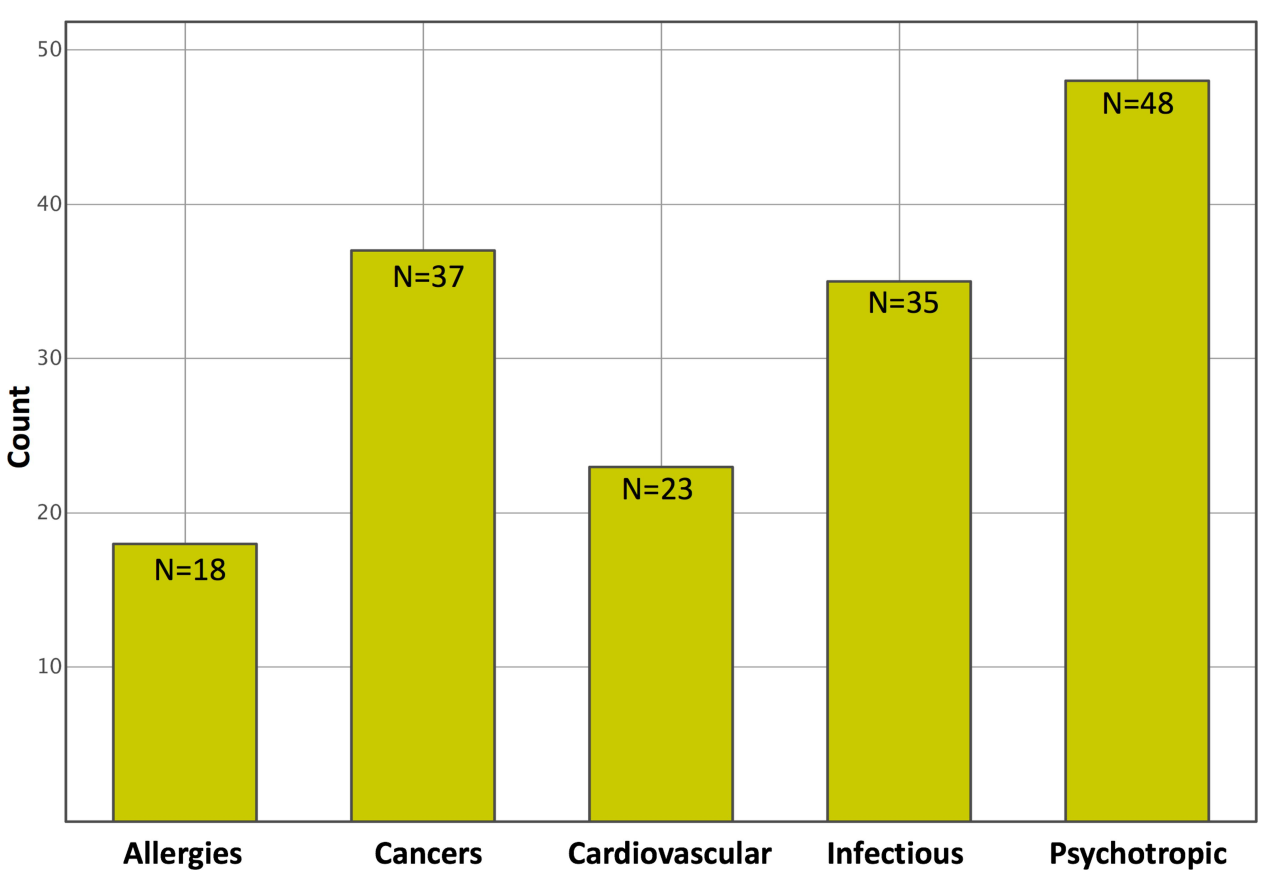

Figure I Five major drug indications found to have SARS-CoV-2 cytopathic effect.

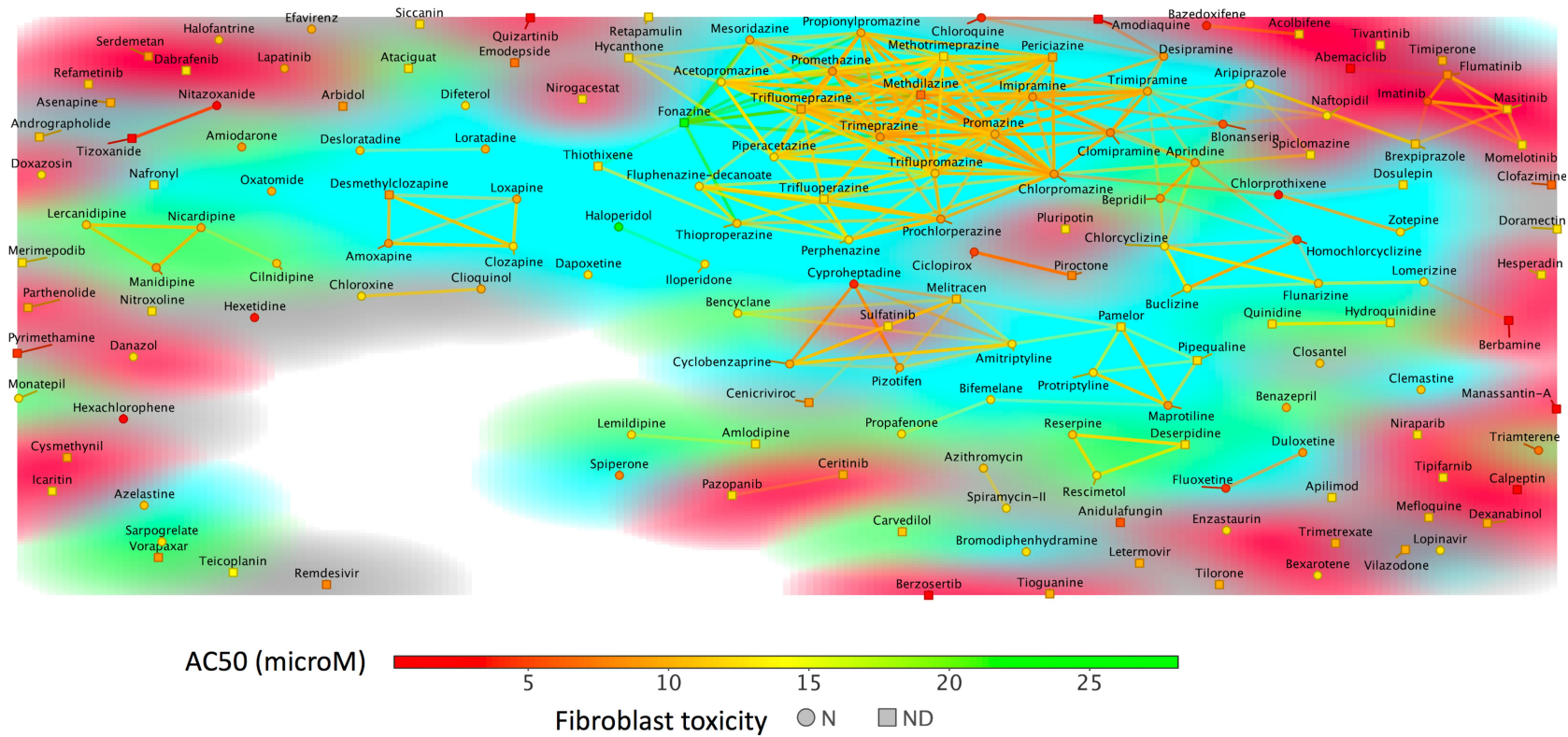

Allergy (antihistamine) and Psychotropic drugs

Cardiovascular diseases

Cancers

Infectious diseases

Figure 2 Bioactive compounds ( 161 molecules) clustered according to the presence of organic functional groups. The molecules that were found to share identical or similar organic functions are connected by lines (eg, the presence of a specific ring system). Increased transparency of the lines reflects decreasing similarity. The compounds are marked either as a circle (molecules not toxic in human fibroblast toxicity assay) or as a square (molecules not tested for such toxicity). The marker symbols and the connecting lines are color coded according to the reported experimental potency values (for this dataset, AC50 values are provided). The background colors behind the markers and lines are color coded according to the disease categories. Yet, as some compounds in the category of "Allergies" are also prescribed in psychiatric settings (indeed some psychotropic drugs were initially used as antihistaminics), we applied the same background color for these two categories to facilitate the reading of this figure. We can observe in this figure a large group of compounds sharing similar substructures (eg, around Promethazine) that have in general moderate activity in these in vitro assays. The figure was generated using DataWarrior version 5.2.1. 
vomiting). While not an antihistamine molecule, Colchicine (approved by the FDA for the management of gout) could be present in this group (although it also has indications in Cancers and Cardiovascular diseases). It is an anti-inflammatory drug that could reduce the risk of complications and death from COVID-19 (study of about 4,500 non-hospitalized patients with COVID-19, the odds of death or hospitalization were apparently $21 \%$ lower in the group on Colchicine). ${ }^{38}$ This molecule is not present in our list of 161 compounds because it was not active in the CPE assays that we investigated and was cytotoxic (ie, indeed this compound can be relatively toxic, depending on the dose). As such, this molecule could possibly act on the exaggerated inflammatory response seen in some patients in more advanced stages of disease progression.

Another group of drugs is anti-cancer agents. Many of these compounds are kinase inhibitors, such as Abemaciclib, Pazopanib, Ceritinib, Lapatinib and Imatinib. Imatinib, a potent ABL inhibitor, was found here to have moderate CPE activity. This compound is in a clinical trial for COVID-19 but other authors noted that this molecule had limited activity on SARS-CoV-2 entry/ infection in different in vitro assay types. ${ }^{39}$ It is also important to note that some kinase inhibitors are used for the treatment of autoimmune disorders and other complex imbalances of the immune system like for instance, Baricitinib. This compound is used for the treatment of rheumatoid arthritis and is in a clinical trial for treating COVID-19 patients. Some authors suggested that it could be beneficial in preventing virus infectivity via perturbation of endocytosis ${ }^{40}$ and/or could be important as an inhibitor of targets in critically ill COVID-19 patients. $^{41}$ This compound is obviously not present in our list of anticancer agents and was not found active in the CPE assay. Yet, such a molecule could be of interest, for instance to act on the cytokine storm. Also, in the cancer field, Pralatrexate was found able to potently inhibit SARS$\mathrm{CoV}-2$ replication with a stronger inhibitory activity than Remdesivir. ${ }^{42}$ It is not present in our list as was found very cytotoxic in the in vitro assays that we analyzed.

Several drugs prescribed in the field of cardiovascular diseases were also found active in the CPE assay. These include Vorapaxar (reduces the incidence of thrombotic cardiovascular events or with peripheral arterial disease, a molecule proposed as potentially interesting for the treatment of COVID-19 patients ${ }^{43}$ ), Carvedilol (treatment of heart failure, hypertension, a molecule also suggested in the literature as potentially beneficial for COVID-19 patients via several mechanisms of action ${ }^{44}$ ) or Nicardipine (antihypertensive properties effective in the treatment of angina and coronary spasms). Another very interesting compound is Bepridil, a long-acting calciumblocking agent with significant anti-anginal activity. This molecule is within a large network of compounds that share structural similarities and is easily identified with simple chemoinformatics visualization approaches (eg, part of the large Promazine-like compounds, see Figure 2). In fact, it is a CAD that can potentially raise the endosomal $\mathrm{pH}$ and that was found highly active in vitro and in animal models against different types of viral infection including Ebola. ${ }^{45}$ This molecule has now been shown, after docking computations and experimental validations, to be potent against SARS-CoV-2 in vitro. ${ }^{46} \mathrm{It}$ is active on the virus main protease and most likely acts on endocytosis and, as such, could interfere with the entry of SARS-CoV-2 into mammalian host cells and slow down replication.

The molecules that belong to the "Infectious Disease" category include molecules with widely different types of activities. For instance, Mefloquine and Chloroquine (antiparasitics for Malaria), Letermovir (for prophylaxis of cytomegalovirus infection), Cenicriviroc (under clinical trials for the treatment of HIV-infection/AIDS), Nitazoxanide (broad-spectrum anti-infective drug) and Lopinavir (in the treatment of HIV-infection). Several antimalarial drugs (eg, Methylene blue, a cationic dye with a tricyclic phenothiazine-like group (in fact a benzene fused to a thiazine ring), that was used as lead for the development of Chlorpromazine and tricyclic antidepressants, approved by the FDA for many indications including the prevention of urinary tract infections in elderly patients and was used during many years to treat malaria) are known to act in vitro on SARS-CoV- $2^{47}$ while the in vitro activity of Cenicriviroc has also been reported. ${ }^{48}$ In the present study, however, Methylene blue was not kept in our final list of 161 compounds as the molecule was reported to be cytotoxic in the NCATS COVID-19 results. This molecule is however interesting mechanistically as in vitro it was found to block (low micromolar in a proteinbased ELISA setup) the Spike-ACE2 protein-protein. ${ }^{49}$ Such a compound is also likely to interact with cell membranes and seems to be a phospholipidosis inducer. ${ }^{50}$ Nitazoxanide was, for example, found promising in its ability to hinder the replication of a SARS-CoV-2 isolate ${ }^{51}$ and is now in several clinical trials either alone (eg, $\mathrm{se}^{52}$ ), against placebo, or in combination with for instance 
Ivermectin (a broad-spectrum anti-parasite medication that was not found to be active in the CPE assays that we investigated here and that was highly cytotoxic in the Vero E6 cell counterscreen, not mentioning potential risks due to interactions or the lack of interactions with some drug transporters in some patients ${ }^{53}$ ). Another wellknown molecule in this category is Azithromycin (an antibiotic medication), which appears to block the binding of the virus to the ACE2 receptor, among others but that was not confirmed as active in various clinical trials, with or without Hydroxychloroquine. It was also found that in 84 elderly patients with suspected COVID-19 treated early with antihistamines (Dexchlorpheniramine, Cetirizine or Loratadine) combined with Azithromycin for the 25 symptomatic cases, could be favorable when monitoring different types of endpoint such as the fatality rate or hospital admission rate. ${ }^{54}$ No adverse effects, no hospital admissions and no deaths were reported. Of course, clinical trials would be needed, but this observation is of interest in the context of the present analysis. The synthetic antibacterial drug Clofoctol, suggested to have activity on SARS-CoV-2, ${ }^{55}$ is not present in our analysis as not tested in this dataset. A molecule with some similarity, Hexachlorophene, a chlorinated bisphenol antiseptic is present in our list and has some in vitro activity but has also some moderate cytotoxic issues. Hexachlorophene was however inactive when tested for human fibroblast toxicity.

Molecules of the "Psychotropic drugs" category also involve structurally diverse compounds that include, for instance, Clomipramine (an antidepressant, also reported in a very recent study ${ }^{56}$ ), Chlorpromazine (an antipsychotic that has activity in vitro and is in clinical trial ${ }^{57-60}$ ), Fluoxetine (an antidepressant, the so-called Prozac), ${ }^{61}$ and Haloperidol (an antipsychotic).

Following the above analysis, we then clustered the small molecules based on their chemical structures. Many different approaches can be used for this purpose ${ }^{62}$ but as the bioactive compounds investigated here tend to belong to a limited number of chemical classes, a possible way is to group the compounds based on the presence of identi$\mathrm{cal} /$ similar organic functional groups. We investigated our list of 161 molecules using this strategy with the DataWarrior application. The results are shown in Figure 2. As can be seen, a large cluster involves drugs used for allergies and for mental disorders. These molecules all share a phenothiazine group with different types of side chains (molecules around Trimeprazine also named
Alimemazine or around Promazine or Fluphenazine, a piperazine ring phenothiazine). Other molecules that are chemically closely related involve for instance Clomipramine or Imipramine. Here, the phenothiazine group is not present but a dibenzazepine group is found instead. With such a simple data visualization method, it becomes possible in one single figure to highlight molecules that could be of interest, along this line, the case of the cardiovascular Bepridil drug mentioned above is of interest.

Earlier we suggested that cationic amphiphilic psychotropic and antihistamine drugs might play a significant protective role as witnessed by the relatively low representation of the psychiatric patients among the COVID-19 clinical units. $^{2}$ The notion of CADs and/or lysosomotropic active agents is not clearly defined but in general, the scientific community considers that the cationic character of a molecule can be flagged by the (predicted) value of its highest basic pKa while the amphiphilicity can be estimated by (computed) $\log$ P values. ${ }^{14,50}$ Such compounds contain organic bases with basic pKa above 6 or 7 and are expected to carry one or more positive charges at physiological $\mathrm{pH}$ (even more so in the acidic environment of the endosomal-lysosomal system) while the computed $\log \mathrm{P}$ value of these molecules is in general above 3. If we apply such criteria to our list of 161 molecules, 125 compounds have a computed $\log$ P above 3 and among these, 105 have at least one positive charge, suggesting that about $65 \%$ of the compounds could be CADs. Obviously these global physicochemical properties are present in a variety of drugs that act in many therapeutic areas. Here we found such compounds in all the five disease categories reported in Figure 1.

From our analysis, the chemical structures or substructures of some CAD compounds that would seem interesting to interfere with SARS-CoV-2 infection are illustrated in Figure 3. According to the automated structural classification package ClassyFire, the main chemical classes involve molecules that contain, as a core, a phenothiazine group (eg, Promazine), a benzazepine group (eg, Imipramine), a benzodiazepine group (eg, Clozapine), a dibenzocycloheptene group (eg, Cyproheptadine), a benzocycloheptapyridine group (eg, Desloratadine), a diazanaphthalene group (eg, Azelastine) or a benzene and substituted derivative group (eg, Homochlorcyclizine or Buclizine, in this case, a piperazine ring is present in the core region). Of importance, other $\mathrm{CAD}$ molecules used in different therapeutic areas could be valuable but as 


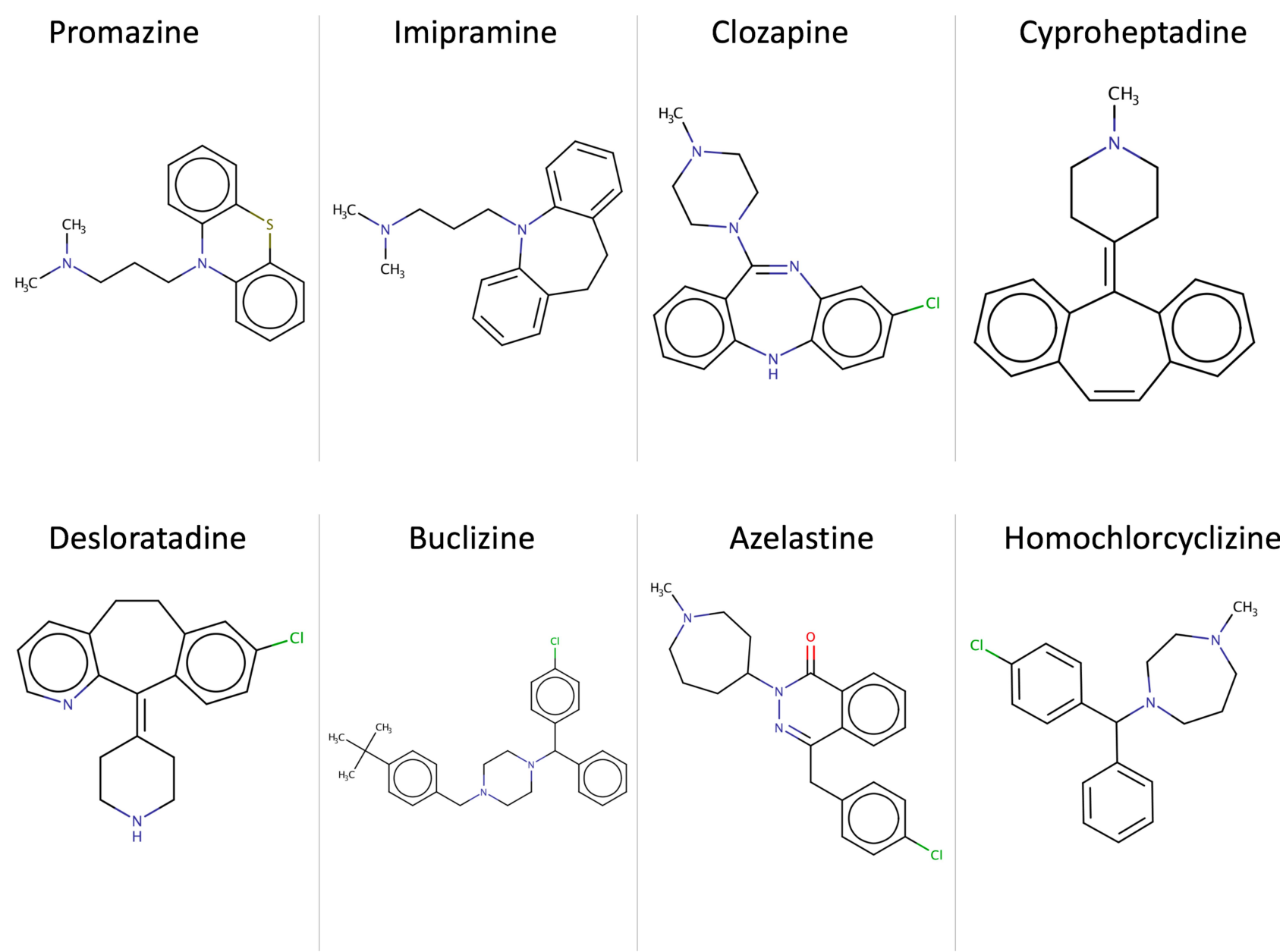

Figure 3 The types of CADs that could interfere with the SARS-CoV-2 life cycle in vitro following our clinical and chemoinformatics analysis. These molecules have a hydrophobic-aromatic ring system and a side chain that carries one (or more) ionizable amine functional group(s). The largest family of molecules contain a phenothiazine group (eg, Promazine, 16 molecules have such a group). The figure was generated using MarvinView from the ChemAxon suite.

compared to cationic amphiphilic psychotropic and antihistamine drugs, we have no clinical observations suggesting a possible protective effect, yet, in vitro studies definitively suggest that these compounds are of interest.

In vitro data can give some insights about the potential of a molecule but cannot predict if a molecule is going to be active in humans. Along the same line, most (small) animal models to study such type of virus tend also to be of limited values to select compounds as they do not reproduce the complexity of the human body. ${ }^{63}$ But, interestingly, after mining 219,000 digital health records, Reznikov et $\mathrm{al}^{64}$ have reported that prior usage of Loratadine, Diphenhydramine, Cetirizine, Hydroxyzine or Azelastine was associated with a reduced incidence of positive SARS-CoV-2 test results in individuals of 61 years and above. Loratadine and Azelastine are present in our list of 161 drugs. Loratadine and Azelastine were also found in other reported experimental screening data available at the open NCATS portal to perturb virus entry via modulation of the ACE2-Spike protein interaction (similar results in this assay were apparently not found for Desloratadine). Diphenhydramine was not tested in the data that we downloaded from the NCATS portal but the highly similar molecule Bromodiphenhydramine was found active in the CPE assay. Yet, Reznikov et al reported that Diphenhydramine was indeed active in vitro. Hydroxyzine and its active metabolite, Cetirizine, were not found active in the CPE assays that we analyzed nor in other assays reported at the open NCATS portal but Hydroxyzine was found to impede the interaction between the viral Spike protein and the human receptor ACE2 and as such could interfere with virus entry (action at the level of the Spike could also be of importance for SARS-CoV-2 virulence). In our hands, Hydroxyzine has only moderate in vitro activity (unpublished data) but we listed it as a very interesting $\mathrm{CAD}^{2}$ and so other authors. ${ }^{9}$ In fact, 
clinical observations suggest that Hydroxyzine could reduce mortality in patients hospitalized for COVID-19 most likely due to the anti-inflammatory properties of the compound. ${ }^{3}$ These data suggest that additional investigations are needed for this compound to fully understand the molecular mechanisms at play and fully assess its activity on the virus and on the host. Along the same line and with the idea of drug combination, it seems possible that the use of two anti-histamine molecules, Cetirizine and Famotidine (not predicted to be CAD), both found to be inactive in the analyzed CPE assays, might be a safe and effective approach to reduce the severity of COVID-19, presumably via a modulation of the histamine-mediated cytokine storm. ${ }^{65}$ Again, one has to be cautious with in vitro data and statistics performed on electronic health records, yet, the data reported above definitively suggest that some of these CAD molecules could be of interest.

Overall, the present analysis, together with other studies, ${ }^{4,9,66-72}$ supports our initial observation ${ }^{2}$ and suggests that some psychiatric patients could have been protected by their pharmacological treatments. Along this line of reasoning, it should also be mentioned that these patients receive in general a cocktail of drugs (psychotropic and anti-histamine molecules) including several CAD compounds possibly working on different molecular mechanisms. Our analysis also highlights some interesting CADs that act on other types of diseases, such as cardiovascular diseases.

\section{Conclusions}

From the above analysis, we suggest that some antihistamine and psychotropic CADs could protect humans from SARS-CoV-2 infection while some of these drugs might be also beneficial at more advanced stages of the disease. Some CAD molecules may only have moderate anti-viral activity at nontoxic concentrations in vitro but could still be of interest (eg, combined with other treatments). The molecular mechanisms involved are likely to be complex, from perturbation of endocytosis, membrane fusion, inhibition of catalytic site and/or of protein-protein interactions to direct binding to some specific membrane receptors. For example, a recent investigation applied surface plasmon resonance to study some antipsychotic drugs and reported that several compounds could bind directly to ACE2 and partially inhibit this virus entry mechanism. ${ }^{73}$ It is of course important to note that psychotropic compounds can induce side effects and might not be safe enough to be prescribed for prophylaxis to a population not concerned with psychiatric issues.
Yet, with regard to SARS-CoV-2 infection, it would seem possible to replace some psychotropic CADs with antihistamine CADs. For prevention purposes, it should also be mentioned that even molecules with moderate in vitro activity might be relevant, while it is also known that, if needed, most antihistamine CADs could be tolerated at higher doses than usually prescribed without inducing severe side effects. Drug combinations could be beneficial, even more so for drugs that have low to moderate toxicity ${ }^{74}$ and usually for compounds that act on different targets and mechanisms. We anticipate many interesting results in this area, for example, the recently demonstrated activity of three drugs (not tested in the dataset investigated here): Clomiphene (selective estrogen receptor modulator and a non-steroidal fertility medicine for women), Vortioxetine (an antidepressant drug), and Asenapine (an atypical antipsychotic drug). All three molecules are, according to our computations, CAD compounds, and in vitro, it seems that the combination is much more potent than each compound used alone. ${ }^{75}$ Obviously, further investigations are needed to evaluate the potential additivity, synergy and hopefully not antagonism of the CADs discussed above. Yet, if active in humans, some of these molecules (or a combination of compounds) may help to face the emergence of variants or the rapid decline of neutralizing antibodies. ${ }^{76-78}$ We believe that clinical trials are now needed to clarify the type of patients that could benefit from these drugs and at which stage of the infection they should be given, prophylaxis being a potential option. Indeed, many medical practitioners and scientists have urged the authorities to consider some antihistamine agents for clinical trials, at least in France, but so far, nothing has apparently been decided. While vaccines are of very high importance, the many drawbacks (manufacturing capabilities, shipping and storage conditions, public acceptance, time to administrate the drug to billions of people, risks with variants ...) call for alternative and/or complementary treatments and preventive approaches in the form of safe, orally available, small molecules that target, if possible, mechanisms not prone to drug resistance.

\section{Acknowledgments}

We thank the foundation FondaMental and INSERM for their supports.

\section{Disclosure}

The authors declare that they have no conflicts of interest for this work nor any competing financial interests or 
personal relationships that could influence the work reported in this paper.

\section{References}

1. Edwards A. What are the odds of finding a COVID-19 drug from a lab repurposing screen? J Chem Inf Model. 2020;60(12):5727-5729. doi:10.1021/acs.jcim.0c00861

2. Villoutreix BO, Beaune PH, Tamouza R, Krishnamoorthy R, Leboyer M. Prevention of COVID-19 by drug repurposing: rationale from drugs prescribed for mental disorders. Drug Discov Today. 2020;25 (8):1287-1290. doi:10.1016/j.drudis.2020.06.022

3. Hoertel N, Sanchez M, Vernet R, et al. Association between hydroxyzine use and reduced mortality in patients hospitalized for coronavirus disease 2019: results from a multicenter observational study medRxiv. 2020

4. Hoertel N, Sánchez-Rico M, Vernet R, et al. Association between antidepressant use and reduced risk of intubation or death in hospitalized patients with COVID-19: results from an observational study. Mol Psychiatry. 2021. doi:10.1038/s41380-021-01021-4

5. Hoertel N, Sánchez-Rico M, Vernet R, et al. Observational study of chlorpromazine in hospitalized patients with COVID-19. Clin Drug Investig. 2021;41:221-233. doi:10.1007/s40261-020-00975-7

6. Ekins S, Mottin M, Ramos P, et al. Déjà vu: stimulating open drug discovery for SARS-CoV-2. Drug Discov Today. 2020;25(5):928941. doi:10.1016/j.drudis.2020.03.019

7. Salata C, Calistri A, Parolin C, Baritussio A, Palù G. Antiviral activity of cationic amphiphilic drugs. Expert Rev Anti Infect Ther 2017;15(5):483-492. doi:10.1080/14787210.2017.1305888

8. Johnson M, Lajiness M, Maggiora G. Molecular similarity: a basis for designing drug screening programs. Prog Clin Biol Res. 1989;291:167-171.

9. Norinder U, Tuck A, Norgren K, Munic Kos V. Existing highly accumulating lysosomotropic drugs with potential for repurposing to target COVID-19. Biomed Pharmacother. 2020;130:110582. doi:10.1016/j.biopha.2020.110582

10. Gies V, Bekaddour N, Dieudonné Y, et al. Beyond anti-viral effects of chloroquine/hydroxychloroquine. Front Immunol. 2020;11:1409. doi:10.3389/fimmu.2020.01409

11. Hathout RM, Abdelhamid SG, Metwally AA. Chloroquine and hydroxychloroquine for combating COVID-19: investigating efficacy and hypothesizing new formulations using bio/chemoinformatics tools. Inform Med Unlocked. 2020;21:100446. doi:10.1016/j.imu.2020.100446

12. Patil VM, Singhal S, Masand N. A systematic review on use of aminoquinolines for the therapeutic management of COVID-19: efficacy, safety and clinical trials. Life Sci. 2020;254:117775. doi:10.1016/j.1fs.2020.117775

13. Savarino A, Boelaert JR, Cassone A, Majori G, Cauda R. Effects of chloroquine on viral infections: an old drug against today's diseases? Lancet Infect Dis. 2003;3(11):722-727. doi:10.1016/S1473-3099(03) 00806-5

14. Blaess M, Kaiser L, Sauer M, Csuk R, Deigner HP. COVID-19/ SARS-CoV-2 infection: lysosomes and lysosomotropism implicate new treatment strategies and personal risks. Int J Mol Sci. 2020;21 (14):4953. doi:10.3390/ijms21144953

15. Breiden B, Sandhoff K. Emerging mechanisms of drug-induced phospholipidosis. Biol Chem. 2019;401(1):31-46. doi:10.1515/hsz2019-0270

16. Ballout RA, Sviridov D, Bukrinsky MI, Remaley AT. The lysosome: a potential juncture between SARS-CoV-2 infectivity and NiemannPick disease type C, with therapeutic implications. FASEB J. 2020;34 (6):7253-7264. doi:10.1096/fj.202000654R

17. Varalda M, Antona A, Bettio V, et al. Psychotropic drugs show anticancer activity by disrupting mitochondrial and lysosomal function. Front Oncol. 2020;10:562196. doi:10.3389/fonc.2020.562196
18. Shang J, Wan Y, Luo C, et al. Cell entry mechanisms of SARS-CoV2. Proc Natl Acad Sci U S A. 2020;117(21):11727-11734. doi:10.10 73/pnas.2003138117

19. Gordon DE, Jang GM, Bouhaddou M, et al. A SARS-CoV-2 protein interaction map reveals targets for drug repurposing. Nature. 2020;583(7816):459-468. doi:10.1038/s41586-020-2286-9

20. Eldanasory OA, Eljaaly K, Memish ZA, Al-Tawfiq JA. Histamine release theory and roles of antihistamine in the treatment of cytokines storm of COVID-19. Travel Med Infect Dis. 2020;37:101874. doi:10.1016/j.tmaid.2020.101874

21. Xu J, Xue Y, Zhou R, Shi PY, Li H, Zhou J. Drug repurposing approach to combating coronavirus: potential drugs and drug targets. Med Res Rev. 2020. doi:10.1002/med.21763

22. Brimacombe KR, Zhao T, Eastman RT, et al. An OpenData portal to share COVID-19 drug repurposing data in real time. bioRxiv. 2020. doi:10.1101/2020.06.04.135046

23. Kim S, Chen J, Cheng T, et al. PubChem in 2021: new data content and improved web interfaces. Nucleic Acids Res. 2021;49:D1388D13895.

24. Mendez D, Gaulton A, Bento AP, et al. ChEMBL: towards direct deposition of bioassay data. Nucleic Acids Res. 2019;47(D1):D930d940. doi:10.1093/nar/gky1075

25. Wishart DS, Feunang YD, Guo AC, et al. DrugBank 5.0: a major update to the DrugBank database for 2018. Nucleic Acids Res. 2018;46(D1):D1074-d1082. doi:10.1093/nar/gkx1037

26. Avram S, Bologa CG, Holmes J, et al. DrugCentral 2021 supports drug discovery and repositioning. Nucleic Acids Res. 2021;49: D1160-D1169.

27. Douguet D. Data sets representative of the structures and experimental properties of FDA-approved drugs. ACS Med Chem Lett. 2018;9 (3):204-209. doi:10.1021/acsmedchemlett.7b00462

28. Sud M. MayaChemTools: an open source package for computational drug discovery. J Chem Inf Model. 2016;56(12):2292-2297. doi:10. 1021/acs.jcim.6b00505

29. Lagorce D, Bouslama L, Becot J, Miteva MA, Villoutreix BO. FAFdrugs4: free ADME-tox filtering computations for chemical biology and early stages drug discovery. Bioinformatics. 2017;33(22):36583660. doi:10.1093/bioinformatics/btx491

30. Shockley KR. Quantitative high-throughput screening data analysis: challenges and recent advances. Drug Discov Today. 2015;20(3):296300. doi: $10.1016 /$ j.drudis.2014.10.005

31. Huang R, Xu M, Zhu H, et al. Massive-scale biological activity-based modeling identifies novel antiviral leads against SARS-CoV-2. bioRxiv. 2020.

32. Ma C, Hu Y, Townsend JA, et al. Ebselen, Disulfiram, Carmofur, PX12, Tideglusib, and Shikonin are nonspecific promiscuous SARSCoV-2 main protease inhibitors. ACS Pharmacol Transl Sci. 2020;3 (6):1265-1277. doi:10.1021/acsptsci.0c00130

33. Lagorce D, Douguet D, Miteva MA, Villoutreix BO. Computational analysis of calculated physicochemical and ADMET properties of protein-protein interaction inhibitors. Sci Rep. 2017;7:46277. doi:10.1038/srep46277

34. Singh N, Chaput L, Villoutreix BO. Virtual screening web servers: designing chemical probes and drug candidates in the cyberspace. Brief Bioinform. 2020.

35. López-López E, Bajorath J, Medina-Franco JL. Informatics for chemistry, biology, and biomedical sciences. J Chem Inf Model. 2021;61 (1):26-35. doi:10.1021/acs.jcim.0c01301

36. Sander T, Freyss J, von Korff M, Rufener C. DataWarrior: an opensource program for chemistry aware data visualization and analysis. $J$ Chem Inf Model. 2015;55(2):460-473. doi:10.1021/ci500588j

37. Djoumbou feunang Y, Eisner R, Knox C, et al. ClassyFire: automated chemical classification with a comprehensive, computable taxonomy. J Cheminform. 2016;8:61. doi:10.1186/s13321-016-0174-y

38. Tardif J-C, Bouabdallaoui N, L'Allier PL, et al. Efficacy of colchicine in non-hospitalized patients with COVID-19. Medrxiv. 2021. 
39. Zhao H, Mendenhall M, Deininger MW. Imatinib is not a potent antiSARS-CoV-2 drug. Leukemia. 2020;34(11):3085-3087. doi:10.1038/ s41375-020-01045-9

40. Favalli EG, Biggioggero M, Maioli G, Caporali R. Baricitinib for COVID-19: a suitable treatment? Lancet Infect Dis. 2020;20 (9):1012-1013. doi:10.1016/S1473-3099(20)30262-0

41. Pairo-Castineira E, Clohisey S, Klaric L, et al. Genetic mechanisms of critical illness in Covid-19. Nature. 2021;591(7848):92-98. doi:10.1038/s41586-020-03065-y

42. Zhang H, Yang Y, Li J, et al. A novel virtual screening procedure identifies Pralatrexate as inhibitor of SARS-CoV-2 RdRp and it reduces viral replication in vitro. PLoS Comput Biol. 2020;16(12): e1008489. doi:10.1371/journal.pcbi.1008489

43. Sriram K, Insel PA. Proteinase-activated receptor 1: a target for repurposing in the treatment of COVID-19? Br J Pharmacol. 2020;177(21):4971-4974. doi:10.1111/bph.15194

44. Skayem C, Ayoub N. Carvedilol and COVID-19: a potential role in reducing infectivity and infection severity of SARS-CoV-2. Am J Med Sci. 2020;360(3):300. doi:10.1016/j.amjms.2020.05.030

45. DeWald LE, Dyall J, Sword JM, et al. The calcium channel blocker bepridil demonstrates efficacy in the murine model of marburg virus disease. J Infect Dis. 2018;218(suppl_5):S588-s591. doi:10.1093/ infdis/jiy332

46. Vatansever EC, Yang KS, Drelich AK, et al. Bepridil is potent against SARS-CoV-2 in vitro. Proc Natl Acad Sci U S A. 2021;118(10). doi:10.1073/pnas.2012201118

47. Gendrot M, Andreani J, Boxberger M, et al. Antimalarial drugs inhibit the replication of SARS-CoV-2: an in vitro evaluation. Travel Med Infect Dis. 2020;37:101873. doi:10.1016/j.tmaid.2020.101873

48. Okamoto M, Toyama M, Baba M. The chemokine receptor antagonist cenicriviroc inhibits the replication of SARS-CoV-2 in vitro. Antiviral Res. 2020;182:104902. doi:10.1016/j.antiviral.2020.104902

49. Bojadzic D, Alcazar O, Buchwald P. Methylene blue inhibits the SARS-CoV-2 Spike-ACE2 protein-protein interaction-a mechanism that can contribute to its antiviral activity against COVID-19. Front Pharmacol. 2020;11:600372. doi:10.3389/fphar.2020.600372

50. Muehlbacher M, Tripal P, Roas F, Kornhuber J. Identification of drugs inducing phospholipidosis by novel in vitro data. ChemMedChem. 2012;7(11):1925-1934. doi:10.1002/cmdc.201200306

51. Mostafa A, Kandeil A, AMM Elshaier Y, et al. FDA-approved drugs with potent in vitro antiviral activity against severe acute respiratory syndrome coronavirus 2. Pharmaceuticals. 2020;13(12):443. doi:10. $3390 /$ ph 13120443

52. Rocco PRM, Silva PL, Cruz FF, et al. Early use of nitazoxanide in mild Covid-19 disease: randomised, placebo-controlled trial. Eur Respir J. 2021. doi:10.1183/13993003.03725-2020

53. Telbisz Á, Ambrus C, Mózner O, et al. Interactions of potential antiCOVID-19 compounds with multispecific ABC and OATP drug transporters. Pharmaceutics. 2021;13(1):81. doi:10.3390/pharmaceutics13010081

54. Morán Blanco JI, Alvarenga Bonilla JA, Homma S, Suzuki K, Fremont-Smith P, Villar Gómez de Las Heras K. Antihistamines and azithromycin as a treatment for COVID-19 on primary health care - a retrospective observational study in elderly patients. Pulm Pharmacol Ther. 2021;67:101989. doi:10.1016/j.pupt.2021.101989

55. Bailly C, Vergoten G. A new horizon for the old antibacterial drug clofoctol. Drug Discov Today. 2021. doi:10.1016/j.drudis.2021.02. 004

56. Gorshkov K, Chen CZ, Bostwick R, et al. The SARS-CoV-2 cytopathic effect is blocked by lysosome alkalizing small molecules. ACS Infect Dis. 2020. doi:10.1021/acsinfecdis.0c00349

57. Plaze M, Attali D, Petit AC, et al. Repurposing chlorpromazine to treat COVID-19: the reCoVery study. L'Encephale. 2020;46(3):169172. doi:10.1016/j.encep.2020.05.006

58. Plaze M, Attali D, Prot M, et al. Inhibition of the replication of SARS-CoV-2 in human cells by the FDA-approved drug chlorpromazine. Int J Antimicrob Agents. 2021;53:106274.
59. Stip E, Rizvi TA, Mustafa F, et al. The large action of chlorpromazine: translational and transdisciplinary considerations in the face of COVID-19. Front Pharmacol. 2020;11:577678. doi:10.3389/ fphar.2020.577678

60. Weston S, Coleman CM, Haupt R, et al. Broad anti-coronavirus activity of food and drug administration-approved drugs against SARS-CoV-2 in vitro and SARS-CoV in vivo. J Virol. 2020;94(21). doi:10.1128/JVI.01218-20

61. Schloer S, Brunotte L, Goretzko J, et al. Targeting the endolysosomal host-SARS-CoV-2 interface by clinically licensed functional inhibitors of acid sphingomyelinase (FIASMA) including the antidepressant fluoxetine. Emerg Microbes Infect. 2020;9(1):2245-2255. doi:10.1080/22221751.2020.1829082

62. Muratov EN, Bajorath J, Sheridan RP, et al. QSAR without borders. Chem Soc Rev. 2020;49(11):3525-3564.

63. Datta PK, Liu F, Fischer T, Rappaport J, Qin X. SARS-CoV-2 pandemic and research gaps: understanding SARS-CoV-2 interaction with the ACE2 receptor and implications for therapy. Theranostics. 2020;10(16):7448-7464. doi:10.7150/thno.48076

64. Reznikov LR, Norris MH, Vashisht R, et al. Identification of antiviral antihistamines for COVID-19 repurposing. Biochem Biophys Res Commun. 2021;538:173-179. doi:10.1016/j.bbrc.2020.11.095

65. Hogan IRB, Hogan Iii RB, Cannon T, et al. Dual-histamine receptor blockade with cetirizine-famotidine reduces pulmonary symptoms in COVID-19 patients. Pulm Pharmacol Ther. 2020;63:101942. doi:10.1016/j.pupt.2020.101942

66. Andersen PI, Ianevski A, Lysvand H, et al. Discovery and development of safe-in-man broad-spectrum antiviral agents. Int J Infect Dis. 2020;93:268-276. doi:10.1016/j.ijid.2020.02.018

67. Lenze EJ, Mattar C, Zorumski CF, et al. Fluvoxamine vs placebo and clinical deterioration in outpatients with symptomatic COVID-19: a randomized clinical trial. JAMA. 2020;324(22):2292-2300. doi:10.10 01/jama.2020.22760

68. Javelot H, Petrignet J, Addiego F, et al. Towards a pharmacochemical hypothesis of the prophylaxis of SARS-CoV-2 by psychoactive substances. Med Hypotheses. 2020;144:110025. doi:10.1016/j.mehy.20 20.110025

69. Vaugeois JM. Psychotropics drugs with cationic amphiphilic properties may afford some protection against SARS-CoV-2: a mechanistic hypothesis. Psychiatry Res. 2020;291:113220. doi:10.1016/j.psychres. 2020.113220

70. Otręba M, Kośmider L, Rzepecka-Stojko A. Antiviral activity of chlorpromazine, fluphenazine, perphenazine, prochlorperazine, and thioridazine towards RNA-viruses. A review. Eur J Pharmacol. 2020;887:173553. doi:10.1016/j.ejphar.2020.173553

71. Faria CGF, Weiner L, Petrignet J, et al. Antihistamine and cationic amphiphilic drugs, old molecules as new tools against the COVID19? Med Hypotheses. 2021;148:110508. doi:10.1016/j.mehy.2021. 110508

72. Barh D, Tiwari S, Weener ME, et al. Multi-omics-based identification of SARS-CoV-2 infection biology and candidate drugs against COVID-19. Comput Biol Med. 2020;126:104051. doi:10.1016/j. compbiomed.2020.104051

73. Lu J, Hou Y, Ge S, et al. Screened antipsychotic drugs inhibit SARSCoV-2 binding with ACE2 in vitro. Life Sci. 2021;266:118889. doi:10.1016/j.lfs.2020.118889

74. Sun W, Sanderson PE, Zheng W. Drug combination therapy increases successful drug repositioning. Drug Discov Today. 2016;21(7):11891195. doi:10.1016/j.drudis.2016.05.015

75. Xiong HL, Cao JL, Shen CG, et al. Several FDA-approved drugs effectively inhibit SARS-CoV-2 infection in vitro. Front Pharmacol. 2021;11:609592. doi:10.3389/fphar.2020.609592

76. Awadasseid A, Wu Y, Tanaka Y, Zhang W. SARS-CoV-2 variants evolved during the early stage of the pandemic and effects of mutations on adaptation in Wuhan populations. Int J Biol Sci. 2021;17 (1):97-106. doi:10.7150/ijbs.47827 
77. Villoutreix BO, Calvez V, Marcelin AG, Khatib A-M. In silico investigation of the New UK (B.1.1.7) and South African (501Y. V2) SARS-CoV-2 variants with a focus at the ACE2-spike RBD interface. Int J Mol Sci. 2021;22(4):1695. doi:10.3390/ijms22041695
78. Marot S, Malet I, Leducq V, et al. Rapid decline of neutralizing antibodies against SARS-CoV-2 among infected healthcare workers. Nat Commun. 2021;12(1):844. doi:10.1038/s41467-021-21111-9

\section{Publish your work in this journal}

Advances and Applications in Bioinformatics and Chemistry is an international, peer-reviewed open-access journal that publishes articles in the following fields: Computational biomodelling; Bioinformatics; Computational genomics; Molecular modelling; Protein structure modelling and structural genomics; Systems Biology; Computational
Biochemistry; Computational Biophysics; Chemoinformatics and Drug Design; In silico ADME/Tox prediction. The manuscript management system is completely online and includes a very quick and fair peerreview system, which is all easy to use. Visit http://www.dovepress.com/ testimonials.php to read real quotes from published authors. 